\title{
Atrial Metastasis of Renal Cell Carcinoma: A Rare Presentation
}

\author{
Shilpi Shah ${ }^{\mathrm{a}, \mathrm{f}}$, Poornima Vinod ${ }^{\mathrm{b}, \mathrm{f}}$, Mohamed Khayata ${ }^{\mathrm{b}}$, Jason L. Lane ${ }^{\mathrm{c}}$, \\ Vinayak Hegde ${ }^{\mathrm{d}}$, Rupesh Raina ${ }^{\mathrm{e}, \mathrm{g}}$
}

\begin{abstract}
Renal cell carcinoma (RCC) is an aggressive and lethal tumor that has a high frequency of metastatic spread to unpredictable sites. One quarter of patients have either distant metastases or significant localregional disease with atypical symptoms on presentation. We present a 41-year-old patient with symptoms of right heart failure and was found to have metastatic renal cell carcinoma with enhancing tumor from left renal vein up to right atrium.
\end{abstract}

Keywords: Renal cell carcinoma; Right atrial mass; Metastasis; Inferior vena cava

\section{Introduction}

Renal cell carcinoma (RCC) is an aggressive and lethal tumor that constitutes approximately $3 \%$ of all adult malignancies [1]. Typical presentations of RCC include hematuria, flank pain and flank mass in advanced stage [2]. RCC has high frequency of metastatic spread to rare sites with atypical presentations. Most cases of RCC are discovered as incidental findings on imaging studies for other reasons, and the tumor is aggressive enough that $25 \%$ of patients have either distant metastases or local-regional disease with atypical symptoms not related to RCC [2]. Intravascular tumor growth into the renal vein and inferior vena cava reported to be seen in about $15 \%$

Manuscript submitted February 18, 2018, accepted March 6, 2018

aNortheast Ohio Medical University, OH, USA

bepartment of Internal Medicine, Cleveland Clinic Akron General, Akron, $\mathrm{OH}, \mathrm{USA}$

'Department of Pathology, Cleveland Clinic Akron General, Akron, OH, USA ${ }^{\mathrm{d} D e p a r t m e n t}$ of Cardiology, Cleveland Clinic Akron General, Akron, $\mathrm{OH}$, USA

eDepartment of Nephrology, Cleveland Clinic Akron General and Akron Children's Hospital, Akron, OH, USA

${ }_{\mathrm{f}}$ Both are first authors.

gCorresponding Author: Rupesh Raina, Department of Nephrology, Cleveland Clinic Akron General and Akron Children's Hospital, Akron, OH 44307, USA. Email: rraina@akronchildrens.org

doi: https://doi.org/10.14740/cr690w of patients with extension into right atrium in approximately $1 \%$ of cases [2].

\section{Case Report}

A 41-year-old Caucasian male presented with progressive lower extremities swelling for $2-3$ months, exertional shortness of breath with intermittent cough for 1 month and a history of weight gain. There was a history of subjective fever, chills, fatigue, occasional bloody sputum, paroxysmal nocturnal dyspnea, nausea and abdominal bloating. Past history was significant for heart failure with preserved ejection fraction, coronary artery disease; type $2 \mathrm{DM}$ and chronic kidney disease of unknown stage. On presentation, he was afebrile with a blood pressure of 109/90 mm Hg, 2+ pitting edema in bilateral lower extremities and mild abdominal distention. Jugular venous pressure was raised, and fine crackles were heard in both the lung bases. There was also a $2+$ holosystolic murmur at the left lower sternal border (in the third and fourth intercostal space).

Initial workup showed GFR of $37.16 \mathrm{~mL} / \mathrm{min} / 1.7 \mathrm{~m}^{2}$, BUN of $31 \mathrm{mg} / \mathrm{dL}$ and a serum creatinine of $1.99 \mathrm{mg} / \mathrm{dL}$. Complete blood count and comprehensive metabolic panel were normal. Chest X-ray showed borderline cardiomegaly and transthoracic echocardiogram revealed ejection fraction of $65 \%$ with stage 1 diastolic dysfunction, right atrial mass $(4.2 \times$ $5.7 \mathrm{~cm}$ ) that was absent on a previous echocardiogram 1 year
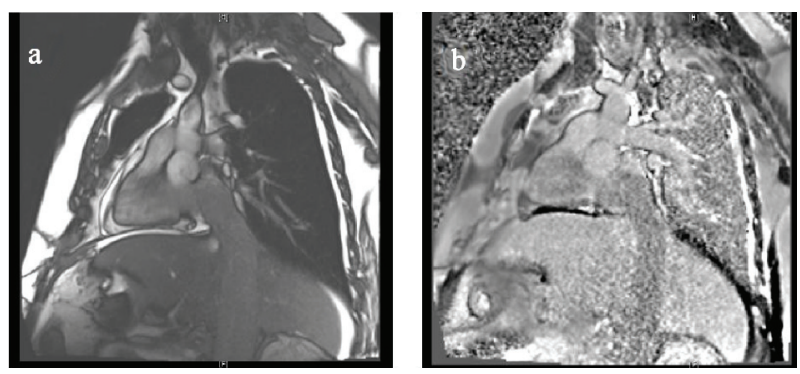

Figure 1. MRI pre and post contrast. (a) and (b): A large oval mass measuring $5 \times 4.2 \mathrm{~cm}$ is visualized within the right atrium. The borders appear to be fairly regular. This mass appears to be in continuity with its extension into the inferior vena cava. A small to medium-sized circumferential pericardial effusion is present without changes of tamponade. The left ventricle is small in size with mild left ventricular hypertrophy. The inferior vena cava is dilated and filled up by echogenic material, likely malignancy. 

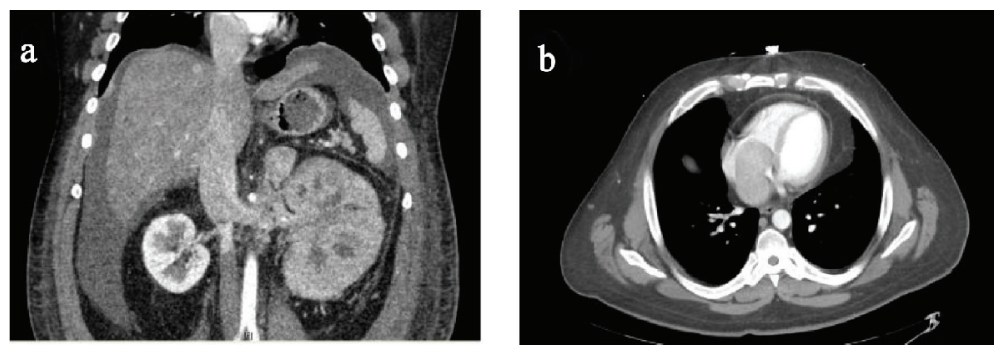

Figure 2. CT chest and abdomen with contrast. (a) and (b): Extensive heterogeneous masslike enlargement of the left kidney, associated with altered areas of cortical thickening/enhancement and architectural distortion. Masslike expansion of the left renal vein and inferior vena cava, extending into the right atrium is suggestive of extensive propagation of enhancing tumor thrombus. Masslike enlargement of the left adrenal gland measuring $3.3 \times 3.2 \mathrm{~cm}$ and multiple small hypervascular nodules throughout the liver, most compatible with metastatic disease.

ago. Cardiac MRI (Fig. 1) showed a large oval mass measuring $5 \times 4.2 \mathrm{~cm}$ within the right atrium with its extension into the inferior vena cava. The left ventricle was small in size with mild left ventricular hypertrophy and normal systolic function with ejection fraction of $55 \%$ and no regional wall motion abnormalities. The inferior vena cava appeared to be dilated and was almost completely filled up by echogenic material, likely malignancy. The findings were most consistent with a metastatic mass within the right atrium.

Contrast CT chest and abdomen (Fig. 2), showed mass in the left kidney, associated with altered areas of cortical thickening/enhancement and architectural distortion. Findings were most suspicious for infiltrating neoplastic involvement, potentially manifestation of renal cell carcinoma. Masslike expansion of the left renal vein and inferior vena cava, extending into the right atrium is suggestive of enhancing tumor thrombus. Left adrenal gland mass $(3.3 \times 3.2 \mathrm{~cm})$ and multiple small hypervascular nodules throughout the liver are most compatible with metastatic disease. Segmental/subsegmental right lower lobe pulmonary embolism was also noted. Urine analysis showed cloudy urine with $100 \mathrm{mg} / \mathrm{dL}$ protein, $5.4 \mathrm{RBC} /$ HPF, 2.6 hyaline casts/LPF, and small amount of hemoglobin. Total free metanephrines, cortisol, renin and aldosterone levels were normal. Biopsy of the adrenal mass (Fig. 3) confirmed the presence of metastatic renal cell carcinoma with papillary features, subtype indeterminate. Final diagnosis was metastatic renal cell carcinoma with papillary features, subtype indeterminate, and right atrial metastasis with right heart failure. Renal vein thrombosis and pulmonary embolism secondary to malignancy induced hypercoagulable state. The patient was treated symptomatically for heart failure, started on a heparin drip and was discharged on apixiban. He was referred to oncology and considered for chemotherapy with temsirolimus.

\section{Discussion}

Renal cell carcinoma typically present with a triad of hematuria, flank pain, and flank mass, especially in cases of advanced disease [2]. In reality however, this classic triad is relatively uncommon, occurring in only $10 \%$ of the cases [3]. Common sites of metastasis include the lungs, adrenals, intestines, brain, and most intra-abdominal organs, but there have been several reported cases of rare metastatic sites [1]. Widespread use of sophisticated imaging modalities has resulted in an increase in the incidental detection of kidney tumors and now more than

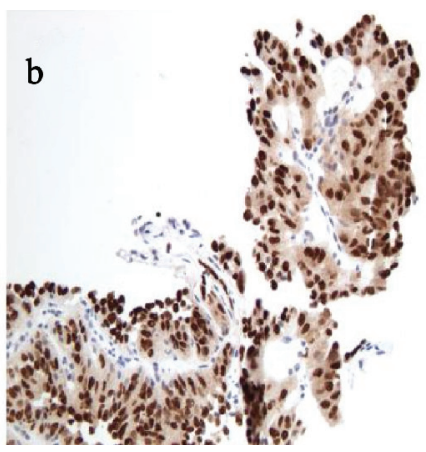

Figure 3. Adrenal biopsy. (a) H\&E stain (100 $\times$ total magnification) and inset (400 $\times$ total magnification). The tumor demonstrates papillary architecture, as evidenced by tumor cells lining fibrovascular cores (arrows). The inset shows tumor cells with abundant eosinophilic cytoplasm, nuclear pleomorphism and mitotic activity (arrowhead) and lacking glandular formation. Importantly, morphologic features of clear cell renal cell carcinoma are not identified. (b) Adrenal biopsy, Pax-8 immunohistochemical stain (200 $\times$ total magnification). Pax-8 is a nuclear transcription factor, which labels tissue of renal, thymic and thyroid origin and is therefore useful for identifying metastatic tumors from these sites. In the clinical context of a patient with a large renal mass, the strong nuclear positivity (brown staining of the tumor cell nuclei) is supportive of origin of this metastatic tumor from a primary renal cortical neoplasm. 
$70 \%$ of all renal cancers are discovered as incidental findings on imaging studies [1]. Our patient presented with symptoms of heart failure, and the diagnosis of RCC was only considered after the imaging studies. Other than primary or metastatic tumors, thrombus and tricuspid valve vegetations, rare causes of a right atrial mass include anatomic variants, coronary fistula, paced wires and indwelling catheters [2]. Renal cell carcinoma has a fascinating feature of invading local vasculature in to the renal vein and grows as a solid column of cells that can sometimes extend up to, as in our patient, the right atrium via inferior vena cava [2]. The commonest histological type of RCC is clear cell variety $(70-80 \%)$, followed by the papillary type $(10 \%)$ [1]. Oftentimes tumors have mixed characteristics of multiple subtypes of RCC, rather than being one distinct cell type [4]. The tumor in our case stained positive for Pax-8 (Fig. 3 ), a finding that is supportive of origin from a primary renal cortical neoplasm. However, the tumor did not show classic morphologic features of any of the common subtypes of renal cell carcinoma and the results of a panel of ancillary immunohistochemical stains were inconclusive as to a specific subtype. The possible surgical option for metastatic RCC extending into the right atrium and causing pulmonary embolism like in our patient is cardiopulmonary bypass (CBP) with deep hypothermal circulatory arrest (DHCA), which is safe and efficient method for removal of the tumor and thrombus [5]. Long-term prognosis of metastatic RCC is poor and survival is limited to months even with the availability of immunotherapy and mTOR kinase inhibitors [1].

\section{Conclusions}

Renal cell carcinoma typically metastasizes to unpredictable sites and presents with atypical symptoms. Classic presentation of RCC is rare and majority of cases are diagnosed as incidental findings on imaging studies for other reasons. High index of suspicion is required to diagnose $\mathrm{RCC}$ with atypical presentations.

\section{Consent}

Informed consent was obtained from the patient for publication.

\section{Conflict of Interest}

The authors have no conflict of interest to declare.

\section{Funding}

This research received no specific grant from any funding agency in the public, commercial, or not-for-profit sectors.

\section{References}

1. Sountoulides P, Metaxa L, Cindolo L. Atypical presentations and rare metastatic sites of renal cell carcinoma: a review of case reports. J Med Case Rep. 2011;5:429.

2. Lu HT, Chong JL, Othman N, Vendargon S, Omar S. An uncommon and insidious presentation of renal cell carcinoma with tumor extending into the inferior vena cava and right atrium: a case report. J Med Case Rep. 2016;10(1):109.

3. Doshi D, Saab M, Singh N. Atypical presentation of renal cell carcinoma: a case report. J Med Case Rep. 2007;1:26.

4. Higgins JP, Rouse RV. Papillary renal cell carcinoma. 2011; Available from: http://surgpathcriteria.stanford. edu/kidney/papillary-renal-cell-carcinoma/printable. html.

5. Posacioglu H, Ayik MF, Zeytunlu M, Amanvermez D, Engin C, Apaydin AZ. Management of renal cell carcinoma with intracardiac extension. J Card Surg. 2008;23(6):754-758. 